\title{
The Effects of Foeniculum vulgare Seed Extract on Fertility Results of Assisted Reproductive Technology in Women With Poor Ovarian Response
}

\author{
Mahnaz Yavangi $^{(\mathbb{D}}$, Ilnaz Mohammadi $^{2}$, Shahedeh Khansari ${ }^{3^{*}}{ }^{\mathbb{D}}$, Shirin Moradkhani $^{4}$, Tayebe Artimani ${ }^{5}$, \\ Saeid Heidari Soureshjani ${ }^{6}$
}

\begin{abstract}
Objectives: Foeniculum vulgare due to phytoestrogens is important in the treatment of female sexual dysfunction including infertility. Accordingly, this study was conducted to investigate the effect of the $F$. vulgare seed extract on the fertility results of assisted reproductive technology (ART) in women with a poor ovarian response (POR).

Materials and Methods: In this before-after intervention, 19 infertile women with POR were enrolled by a convenience sampling method. The amounts of luteinizing hormone ( $\mathrm{LH})$ and follicle-stimulating hormone (FSH), ovarian ultrasound volume, the number of preantral follicles, and the size of the prominent ovary were measured before treatment with $F$. vulgare. Then, patients were treated with $F$. vulgare for two months, followed by initiating the in vitro fertilization (IVF) cycle. The number of embryos transferred in previous and current cycles was investigated after IVF. Finally, the data were analyzed in SPSS 16.

Results: There was a significant difference in the serum LH level $(P=0.002), \mathrm{LH} / \mathrm{FSH}(P=0.049)$, the number of follicles and ovules $(P=0.003)$, endometrial thickness $(P=0.04)$, and ovarian volume $(P=0.03)$ between before and after treatment with $F$. vulgare. Moreover, a significant difference regarding the decreased number of required days for induction was observed between before and after treatment with $F$. vulgare $(P=0.022)$.

Conclusions: In general, the use of $F$. vulgare had positive effects on improving the quality of oocytes and female fertility indices in women with POR.

Keywords: Foeniculum vulgare, Fertility, Ovarian failure, Phytotherapy, Assisted reproductive techniques
\end{abstract}

\section{Introduction}

Infertility is considered the main problem of many couples worldwide (1). Female infertility is associated with unpleasant social outcomes (2) and different psychological problems (3).

Ovarian function is the main issue that is addressed in counseling the infertile people who should undergo treatment with different protocols of ovarian stimulation $(4,5)$. However, some women do not respond well to the standard protocol stimulation regimen and cannot recruit adequate follicles. According to (6), these patients are called 'poor ovarian responders (PORs)'. In vitro fertilization (IVF) is one of the most common and relatively new treatments in assisted reproductive technologies (ARTs) and is associated with great success in the treatment of female infertility (7).

However, in some cases, namely, poor responders, the ovary does not respond appropriately to stimulation and ovulation induction. For instance, poor response to ovarian stimulation was reported in $9 \%-24 \%$ of patients who had IVF (8). Moreover, the prevalence rate may have been underestimated because of inadequate assessments (9).

It is necessary to develop specific therapeutic processes and therapeutic strategies to achieve better outcomes in the treatment of ovarian failure. Many women with ovarian failure need to undergo IVF which has a relatively high failure rate; therefore, the development of therapeutic approaches and interventions seems essential (9). The use of herbal drugs and nature-based products is one of the popular treatments that have recently become widespread. This is due to fewer side effects following the use of the plants in the treatment of diseases $(10,11)$.

Fennel (Foeniculum vulgare Mill), which is from family Apiaceae (Umbelliferae), is a perennial plant that occurs in Mediterranean regions. The dried seeds of $F$. vulgare have pleasant taste and odor and are used as odor and taste-producing agents in food industries. In 
addition, F. vulgare odorous seeds are used as anticancer, stimulant, carminative, and anti-inflammatory agents in different organs of the body $(12,13)$.

Based on Iranian traditional medicine, F. vulgare is a medicinal plant that is warm and dry and very effective in regulating female hormones. Further, F. vulgare seeds have certain effects such as improving menstrual cycles, increasing lactation, reducing the symptoms of dysmenorrhea, facilitating childbearing, reducing premenstrual syndrome, and increasing libido in women. Furthermore, they have estrogenic properties which are useful for treating gynecological diseases (14-16). Moreover, it prevents ovarian cysts, breast cysts, ovarian laziness, and infertility related ovarian laziness, all of which are effective in female infertility.

The cycles of treatments for infertility, especially IVF, are costly (17), and due to chemical drug-induced side effects, the therapeutic process of infertility leads to high levels of mental and emotional stress in women (18).

The demographic transition in developing countries and the aging of the population in these countries have led to increased fertility problems. Given the high financial costs of the current methods of infertility treatment and the high rate of failure in advanced ages, it is important to evaluate the efficacy of different therapies on fertility results. Considering the ethnobotanical studies conducted in Iran and the use of $F$. vulgare to treat some of the sexual female disorders in the Iranian traditional medicine $(19,20)$, the present study aimed to evaluate the effect of $F$. vulgare seed extract on fertility results of ARTs in women with a poor ovarian response.

\section{Materials and Methods}

In this before-after intervention, 19 eligible infertile women with POR referring to the Endometrium and Endometriosis Research Center of Hamadan, Iran were enrolled using the convenience sampling technique in April-August 2014. The inclusion criteria were having a history of infertility (at least 3 years) and suffering from POR with at least one of these criteria such as being over 35 years and having follicle-stimulating hormone (FSH) $\geq$ $10 \mathrm{mlU} / \mathrm{mL}$ on day 3 of the cycle, ovarian volume $\geq 3 \mathrm{cc}$, history of failed ART, $<3-5$ ovules, and previous controlled ovarian hyperstimulation. On the other hand, the exclusion criteria included $\mathrm{FSH}>20 \mathrm{mlU} / \mathrm{mL}$ at an early follicular phase (days 2-4), uterine anomaly or fallopian tube obstruction confirmed by sonohysterogram or hysterosalpingogram, the use of corticosteroids within the past three months, any pregnancy contraindications, the absence of an ovary, and affliction with systemic diseases.

In this study, high-performance liquid chromatography (HPLC) was used for the standardization of herbal products. The applied fennel seed was subjected to phytochemical screening in the Department of Pharmacognosy, School of Pharmacy, Hamadan University of Medical Sciences, Hamadan, Iran. Thus, the powdered seeds were subjected to hydrodistillation extraction using a Clevenger-type apparatus. The resulting oil was injected into gas chromatography-mass spectrophotometry. In addition, the compounds were identified based on the retention times of the $n$-alkanes, which were injected after the oil, under the same chromatographic conditions and retention indices with those reported in the literature, as well as by the comparison of their mass spectra with those held in Wiley library of mass spectra or with the published mass spectra. The yield of the obtained oil from the fennel seed was $0.45 \%$. The main component of the oil was anethol, which constitutes $86.86 \%$ of the oil composition. Further, the microbial quality was determined by the standard plate count method, followed by approving their microbiological safety.

At baseline, the medical descriptions of all women with a history of unsuccessful ART were obtained after their referral to the studied center. Women with previous ART were investigated for POR according to their medical records. Then, the required data were gathered by a checklist which consisted of two sections. The first section was concerned with demographic characteristics, a history of infertility, the number of children, parity, previous diseases and surgery, drug-taking, a history of regular or irregular menstruation, and previous IVF.

These data were drawn from the patients' medical files. The second section of this checklist included paraclinical tests such as FSH levels on day 3 of the cycle and luteinizing hormone $(\mathrm{LH})$, ovarian volume, the number of preantral follicles, endometrial thickness, and the number of days required for induction were measured $(21,22)$ before and after treatment with boiled F. vulgare. Then, the serum levels of FSH, LH, and estradiol were measured on day 3 of the cycle, ovarian volume on day 3 of the cycle, and mid-cycle transvaginal ultrasound.

The patients orally took 50-cc, a single dose of boiled $F$. vulgare each night. To prepare the extract, 10 $\mathrm{g}$ of the already prepared and packaged $F$. vulgare by the researchers in each turn was boiled in $250 \mathrm{~mL}$ water for 20 minutes. After the 2-month treatment with F. vulgare, the paraclinical tests conducted at the baseline were repeated and then the patients underwent ovulation induction while they continued to take boiled F. vulgare (23).

To induce ovulation, two decapeptide ampoules (0.1 unit) were administered to the patients on two days and the administration with 300-450 IU gonadotropin was started from the day 3 onwards and transvaginal ultrasound was conducted once every 3-4 days by the researcher under the supervision of a gynecologist in the studied center. This process continued until the follicular size reached $18 \mathrm{~mm}$ when 2 human chorionic gonadotropin (hCG) ampoules (10000 units) were administered to the patients. Thirty-six hours later, the puncture was conducted, followed by conducting microinjection (1-2 hour(s) later) using the processed sperm, transfer (48-72 hours later), and the beta (hCG 
test (2 weeks later) in the laboratory of the hospital.

Eventually, the data were analyzed by the paired $t$ test, as well as Wilcoxon's and McNemar tests in SPSS, version 16 .

\section{Results}

The yield of the obtained oil from the fennel seed was $0.45 \%$ and anethol was the main component of the oil, which constituted $86.86 \%$ of the oil composition (Table 1 ). The mean (SD) age of the studied patients was 36.95 (6.12) within the range of 31-45) years. Table 2 provides further demographic characteristics of the patients. The mean number of the patients' children the mean parity, and the mean number of abortions were $0.59 \pm 0.37,2.08 \pm 1$, and $2.08 \pm 0.68$, respectively.

Wilcoxon test results demonstrated that there was a significant difference in the serum FSH level between before and after treatment with $F$. vulgare $(P=0.501)$. Additionally, the results of the $t$-test indicated a significant difference in the serum LH level between before and after treatment with $F$. vulgare $(P=0.002)$. In addition, a significant difference was observed in the serum FSH/HL level between before and after treatment with $F$. vulgare $(P=0.049)$, the details of which are provided in Table 3 .

Further, there were significant differences in the number of follicles $(P=0.003)$, the number of ovules $(P=0.003)$, and ovarian volume $(P=0.03)$ in the patients between before and after treatment with $F$. vulgare. Based on the results of the Wilcoxon test, a significant difference was found in the number of days required for induction between before and after treatment with

Table 1. The HPLC Analysis of Foeniculum vulgare

\begin{tabular}{ll}
\hline Compound & Area \% \\
\hline$\alpha$-Pinene & 0.06 \\
Sabinene & 0.12 \\
$\beta$-Myrcene & 0.08 \\
p-Cymene & 0.03 \\
Limonene & 0.88 \\
1,8-Cineol & 0.18 \\
Fenchone & 7.43 \\
Camphor & 0.1 \\
p-Allylanisole & 1.65 \\
E-Anethol & 86.86 \\
Thymol & 1.21 \\
Nonadecane & 0.16 \\
Tricosane & 0.2 \\
Tetracosane & 0.24 \\
Pentacosane & 0.35 \\
Bis(2-ethylhexyl)phthalate & 0.45 \\
\hline
\end{tabular}

Note. HPLC: High-performance liquid chromatography.
F. vulgare $(P=0.022)$. However, the results indicated no significant difference in the number of embryos between before and after treatment with $F$. vulgare $(P=0.677)$. Contrarily, the results of the dependent $t$ test (Table 4) demonstrated a significant difference in the endometrial thickness between before and after treatment with $F$. vulgare $(P=0.04)$.

The study of the conception frequency demonstrated that two (10.5\%) patients conceived after treatment with F. vulgare and before ART.

\section{Discussion}

To the best of our knowledge, the present study was the first one to investigate the use of the F. vulgare seed extract on fertility results in women with POR. The results demonstrated that the levels of $\mathrm{LH}$ and $\mathrm{FSH} /$ LH increased in the patients after treatment with $F$. vulgare. This finding represents the effect of $F$. vulgare on the serum FSH/LH level that is an important factor for the prediction of the response to stimulation. However, hormonal response to the serum FSH level was not significantly different between before and after the treatment. In an in vitro study, F. vulgare extract was found to cause an increase in the serum FSH level while a decrease in LH and testosterone levels in laboratory mice (24).

Based on the findings of this study, the number of ovules and follicles, ovarian volume, and endometrial thickness increased whereas the number of days required for induction decreased after treatment with F. vulgare. In a study conducted on laboratory female mice, it was demonstrated that hydroalcoholic F. vulgare seed

Table 2. Frequency Distribution of the Patients According to the History of Infertility, Type of Menstruation, and Previous Surgery

\begin{tabular}{llcc}
\hline Variable & & Number & Percent (100\%) \\
\hline $\begin{array}{llc}\text { History of } \\
\text { infertility }\end{array}$ & Primary & 11 & 57.9 \\
& Secondary & 8 & 42.1 \\
\hline \multirow{2}{*}{ Menstruation } & Regular & 17 & 89.5 \\
& Irregular & 2 & 10.5 \\
\hline \multirow{2}{*}{$\begin{array}{l}\text { Previous } \\
\text { surgery }\end{array}$} & Yes & 5 & 26.3 \\
\hline
\end{tabular}

Table 3. Mean Serum Levels of $\mathrm{LH}, \mathrm{FSH}$, and FSH/LH in the Patients Before and After Treatment With Foeniculum vulgare

\begin{tabular}{lccc}
\hline \multirow{2}{*}{ Variable } & Before Treatment & After Treatment & \\
\cline { 2 - 3 } & Mean \pm SD & Mean \pm SD & \\
\hline FSH & $8.12 \pm 4.27$ & $7.86 \pm 2.65$ & 0.501 \\
LH & $3.54 \pm 1.28$ & $4.05 \pm 1.71$ & 0.002 \\
FSH/LH & $2.48 \pm 1.61$ & $2.51 \pm 0.96$ & 0.049 \\
\hline
\end{tabular}

Note. SD: standard deviation; LH: luteinizing hormone; FSH: folliclestimulating hormone. 
Table 4. Mean Number of Ovules, Embryos, and Follicles, Ovarian Volume, the Number of Days Required for Induction and Endometrial Thickness at Mid-cycle in Patients Before and After Treatment With Foeniculum vulgare

\begin{tabular}{lccc}
\hline Variable & $\begin{array}{c}\text { Before } \\
\text { Treatment } \\
\text { Mean } \pm \text { SD }\end{array}$ & $\begin{array}{c}\text { After } \\
\text { Treatment } \\
\text { Mean } \pm \text { SD }\end{array}$ & $\begin{array}{c}\text { P } \\
\text { Value }\end{array}$ \\
\hline Number of ovules & $5.37 \pm 4.15$ & $5.53 \pm 5.47$ & 0.003 \\
Number of embryos & $2.05 \pm 2.14$ & $1.95 \pm 1.90$ & 0.677 \\
Number of follicles & $2.78 \pm 1.43$ & $4.45 \pm 2.83$ & 0.003 \\
Ovarian volume & $3.55 \pm 1.97$ & $4.15 \pm 2.21$ & 0.03 \\
Required days for induction & $13.29 \pm 2.51$ & $12.64 \pm 2.23$ & 0.022 \\
Endometrial thickness & $7.50 \pm 2.17$ & $8.44 \pm 2.26$ & 0.046 \\
\hline
\end{tabular}

Note. SD: standard deviation.

extract increased the number of antral, graafian, and multilaminar follicles such that the folliculogenesis and growth of ovarian follicles indicated an increase (25).

Obviously, the increased number of preantral follicles after the use of $F$. vulgare extract represents its positive effect on the ovarian reserve. It seems that the F. vulgare seed exerts these effects because it contains certain phytoestrogens such as isoflavones, prenylated flavonoids, and coumestans (26). Furthermore, the estrogenic activity of $F$. vulgare is due to the presence of a compound called "anatole". Trans-anatole comprises over $80 \%$ of F. vulgare (27-29).

This compound causes an increase in the lactation, the relief of menstrual pain, the facilitation of childbearing, primary dysmenorrhea, and a decrease in female infertility $(30,31)$. This is because phytoestrogens act similar to the estrogens in the body and cause the regulation of the gonad-stimulating hormone activity, an increase in the endometrial thickness, and finally, an increase in the likelihood of fertility. Moreover, the antioxidant properties of the $F$. vulgare seed can increase fertility in women with POR through inhibiting cell destruction while increasing cell growth (15,32-34). In addition, low concentrations of the ethanolic F. vulgare seed extract help increase the alkaline phosphatase activity and thus cell growth and proliferation $(31,35)$. Contrarily, the results demonstrated that the number of embryos did not change significantly after the use of $F$. vulgare extract in the studied patients, which could be due to their old age.

However, the success rate of ARTs used for infertile couples depends on several factors such as the selection of an appropriate protocol, the appropriate implementation of ovarian stimulation, the production of an appropriate ovarian response in the hormonal cycle, along with the number of the oocytes. POR in the IVF cycle is associated with a higher failure rate, a lower number of aspirated oocytes and transferred embryos, and therefore, a lower likelihood of conception (36).

In this study, two (10.5\%) patients, who were above 35 years, conceived after the use of the F. vulgare extract and before beginning the IVF (i.e., an ART). It seems that the use of $F$. vulgare is effective in enhancing the oocyte quality and thus increasing fertility likelihood in humans.

Although the results of this study demonstrated the positive effects of F. vulgare on female fertility, the active dose of the extract should be taken into account since the teratogenic side effects of this extract have already been discussed (37). The small sample size and withdrawal of a number of women (6 women) from continuing participation in the study are the limitations of the present study.

\section{Conclusions}

In general, the $F$. vulgare seed extract is effective in improving the factors for conception in women and can be used as a complementary therapy alongside certain approaches such as ARTs. This study can be considered as a pioneering research project regarding investigating the effect of $F$. vulgare on IVF fertility results in women with POR. Future studies are recommended to evaluate the action mechanism of ovulation of the F. vulgare seed and its extract active dose. In addition, similar studies should be conducted to investigate this issue in nonpoor responders while considering age as a confounder.

\section{Conflict of Interests}

The authors declare that they have no conflict of interests.

\section{Ethical Issues}

The patients signed the written informed consent form for participating in the study, and the research purposes were explained to them. Furthermore, the study protocol was registered in the Iranian Registry of Clinical Trials (Identifier: IRCT201411269014N48; https://www.irct.ir/ trial/9487). Moreover, the study protocol was approved by Hamadan University of Medical Sciences Ethics Committee (number: 16.35.9.1540) in accordance with the research ethical standards.

\section{Financial Support}

The present study was supported by the Vice-chancellor for the Research and Technology of Hamadan University of Medical Sciences.

\section{Acknowledgments}

Hereby, we gratefully thank the Research and Technology Deputy of the Hamadan University of Medical Sciences and all people who assisted us in conducting this study.

\section{References}

1. Mascarenhas MN, Flaxman SR, Boerma T, Vanderpoel S, Stevens GA. National, Regional, and Global Trends in Infertility Prevalence Since 1990: A Systematic Analysis of Health Surveys. PLoS Med. 2012;9(12):e1001356. doi:10.1371/journal.pmed.1001356. 
2. Greil AL, Slauson-Blevins K, McQuillan J. The experience of infertility: a review of recent literature. Sociol Health Illness. 2010;32(1):140-62. doi: 10.1111/j.14679566.2009.01213.x.

3. Begum BN, Hasan S. Psychological problems among women with infertility problem: comparative study. J Pak Med Assoc. 2014;64(11):1287-91.

4. Jirge PR. Ovarian reserve tests. J Hum Reprod Sci. 2011;4(3):108-13.

5. Ramalho de Carvalho B, Gomes Sobrinho DB, Vieira ADD, et al. Ovarian Reserve Assessment for Infertility Investigation. ISRN Obstet Gynecol. 2012;2012:576385. doi: $10.5402 / 2012 / 576385$

6. Badawy A, Wageah A, El Gharib M, Osman EE. Prediction and diagnosis of poor ovarian response: the dilemma. J Reprod Infertil. 2011;12(4):241-8.

7. Kamel RM. Assisted Reproductive Technology after the Birth of Louise Brown. J Reprod Infertil. 2013;14(3):96109.

8. Venetis CA, Kolibianakis EM, Tarlatzi TB, Tarlatzis BC. Evidence based management of poor ovarian response. Ann N Y Acad Sci. 2010; 1205:199-206. doi: 10.1111/j.17496632.2010.05665.x.

9. Jirge PR. Poor ovarian reserve. J Hum Reprod Sci. 2016;9(2):63-9.

10. de Souza PA, Palumbo A Jr, Alves LM, et al. Effects of a nanocomposite containing Orbignya speciosa lipophilic extract on Benign Prostatic Hyperplasia. J Ethnopharmacol. 2011;135(1):135-46. doi: 10.1016/j.jep.2011.03.003.

11. Zhang W, Wang X, Liu Y, et al. Effects of dietary flaxseed lignan extract on symptoms of benign prostatic hyperplasia. J Med Food. 2008;11(2):207-14. doi: 10.1089/jmf.2007.602.

12. Chainy GB, Manna SK, Chaturvedi MM, Aggarwal BB. Anethole blocks both early and late cellular responses transduced by tumor necrosis factor: effect on NFkappa B, AP-1, JNK, MAPKK and apoptosis. Oncogene. 2000;19(25):2943-50. doi: 10.1038/sj.onc.1203614

13. Aggarwal BB, Prasad S, Reuter S, et al. Identification of novel anti-inflammatory agents from ayurvedic medicine for prevention of chronic diseases: "reverse pharmacology" and "bedside to bench" approach. Curr Drug Targets. 2011;12(11):1595-653. doi: 10.2174/138945011798109464

14. Rather MA, Dar BA, Sofi SN, Bhat BA, Qurishi MA. Foeniculum vulgare: A comprehensive review of its traditional use, phytochemistry, pharmacology, and safety. Arab J Chem. 2016;9:S1574-S83. doi: 10.1016/j. arabjc.2012.04.011

15. Oktay M, Gülçin İ, Küfrevioğlu Öİ. Determination of in vitro antioxidant activity of fennel (Foeniculum vulgare) seed extracts. Food Sci Technol. 2003;36(2):263-71. doi: 10.1016/S0023-6438(02)00226-8

16. Ostad SN, Soodi M, Shariffzadeh M, Khorshidi N, Marzban $\mathrm{H}$. The effect of fennel essential oil on uterine contraction as a model for dysmenorrhea, pharmacology and toxicology study. J Ethnopharmacol. 2001;76(3):299304.

17. Katz P, Showstack J, Smith JF, et al. Costs of infertility treatment: Results from an 18-month prospective cohort study. Fertil Steril. 2011;95(3):915-21. doi: 10.1016/j. fertnstert.2010.11.026.
18. Hasanpoor-Azghdy SB, Simbar M, Vedadhir A. The emotional-psychological consequences of infertility among infertile women seeking treatment: Results of a qualitative study. Iran J Rep Med. 2014;12(2):131-8.

19. Karampoor P, Azarnia, A, Mahnaz, A Mirabolghasemi, Ghadireh, A, Alizadeh, Farzane. The Effect of Hydroalcoholic Extract of fennel (Foeniculum vulgare) seed on serum levels of sexual hormones in female Wistar rats with polycystic ovarian syndrome (PCOS). Arak Univ Med Sci J. 2014;17(5):70-8.

20. Khazaei M, Montaseri A, Khazaei MR, Khanahmadi M. Study of Foeniculum vulgare Effect on Folliculogenesis in Female Mice. Int J Fertil Steril. 2011;5(3):122-7.

21. Roudebush WE, Kivens WJ, Mattke JM. Biomarkers of Ovarian Reserve. Biomarker Insights. 2008;3:259-68.

22. Lai Q, Chen C, Zhang Z, et al. The significance of antral follicle size prior to stimulation in predicting ovarian response in a multiple dose $\mathrm{GnRH}$ antagonist protocol. Int J Clin Exp Pathol. 2013;6(2):258-66.

23. Bae J, Kim J, Choue R, Lim H. Fennel (Foeniculum vulgare) and fenugreek (Trigonella foenum-graecum) tea drinking suppresses subjective short-term appetite in overweight women. Clin Nutr Res. 2015;4(3):168-74. doi: 10.7762/ cnr.2015.4.3.168.

24. Mosaddegh M, Esmaeili S, Hassanpour A, Malekmohammadi M, Naghibi F. Ethnobotanical study in the highland of Alvand and Tuyserkan, Iran. Iran J Pharm Res. 2016; 3(1):7-17.

25. Dolatkhahi M, Dolatkhahi A, Nejad JB. Ethnobotanical study of medicinal plants used in Arjan-Parishan protected area in Fars Province of Iran. Avicenna J Phytomed. 2014;4(6):402.

26. Shahat AA, Ibrahim AY, Hendawy SF, et al. Chemical composition, antimicrobial and antioxidant activities of essential oils from organically cultivated fennel cultivars. Molecules. 2011;16(2):1366-77. doi: 10.3390/ molecules16021366.

27. Singh G, Maurya S, De Lampasona M, Catalan C. Chemical constituents, antifungal and antioxidative potential of Foeniculum vulgare volatile oil and its acetone extract. Food control. 2006;17(9):745-52. doi: 10.1016/j. foodcont.2005.03.010

28. Nakagawa Y, Suzuki T. Cytotoxic and xenoestrogenic effects via biotransformation of trans-anethole on isolated rat hepatocytes and cultured MCF-7 human breast cancer cells. Biochem Pharmacol. 2003;66(1):63-73. doi:10.1016/ s0006-2952(03)00208-9

29. Tognolini M, Ballabeni V, Bertoni S, Bruni R, Impicciatore M, Barocelli E. Protective effect of Foeniculum vulgare essential oil and anethole in an experimental model of thrombosis. Pharmacol Res. 2007;56(3):254-60. doi:10.1016/j.phrs.2007.07.002

30. Kooti W, Moradi M, Akbari SA, Sharafi-Ahvazi N, Asadi-Samani M, Ashtary-Larky D. Therapeutic and pharmacological potential of Foeniculum vulgare Mill: a review. J HerbMed Pharmacol. 2015;4(1):1-9.

31. Wei YJ, Tsai KS, Lin LC, et al. Catechin stimulates osteogenesis by enhancing PP2A activity in human mesenchymal stem cells. Osteoporos Int. 2011;22(5):146979. doi: 10.1007/s00198-010-1352-9. 
32. Mohamad RH, El-Bastawesy AM, Abdel-Monem MG, et al. Antioxidant and anticarcinogenic effects of methanolic extract and volatile oil of fennel seeds (Foeniculum vulgare). J Med Food. 2011;14(9):986-1001. doi: 10.1089/ jmf.2008.0255.

33. Zhang ZG, Lu XB, Xiao L, et al. [Antioxidant effects of the Uygur herb, Foeniculum vulgare Mill, in a rat model of hepatic fibrosis. Zhonghua Gan Zang Bing Za Zhi. 2012;20(3):2216. doi: 10.3760/cma.j.issn.1007-3418.2012.03.017.

34. Sadrefozalayi S, Farokhi F. Effect of the aqueous extract of Foeniculum vulgare (fennel) on the kidney in experimental PCOS female rats. Avicenna J Phytomed. 2014;4(2):110-7. doi::10.22038/ajp.2014.1824

35. Mahmoudi Z, Soleimani M, saidi A, Khamisipour G, Azizsoltani A. Effects of Foeniculum vulgare ethanol extracts on osteogenesis in human mecenchymal stem cells. Avicenna Journal of Phytomedicine. 2013;3(2):135-42.

36. Surrey ES, Schoolcraft WB. Evaluating strategies for improving ovarian response of the poor responder undergoing assisted reproductive techniques. Fertil Steril. 2000;73(4):667-76. doi:10.1016/s0015-0282(99)00630-5

37. Barilli SLS, Pereira MSL, Foscarini PT, Silva F, Montanari T. An experimental investigation on effect of Foeniculum vulgare Mill. on gestation. Reprod. 2012;1151:27.

(C) 2020 The Author (s); This is an open-access article distributed under the terms of the Creative Commons Attribution License (http://creativecommons.org/licenses/by/4.0), which permits unrestricted use, distribution, and reproduction in any medium, provided the original work is properly cited. 